\title{
Inactivación potencial del coronavirus SARS-CoV2: ¿qué agentes germicidas se proponen?
}

Potential inactivation of SARS-CoV2 coronavirus: which germicidal agents are proposed?

Inativação potencial do coronavírus SARS-CoV2: quais agentes germicidas são propostos?

Como citar este artículo: Wilches Visbal Jorge Homero, Castillo Pedraza Midian Clara, Serpa Romero Xiomara Zilena. Inactivación potencial del coronavirus SARS-CoV2: ¿qué agentes germicidas se proponen?. Revista Cuidarte. 2021;12(1):e1273. http://dx.doi.org/10.15649/cuidarte.1273

Revista Cuidarte Rev Cuid. Ene. - Abril. 2021; 12(1): e1273 doi' http://dx.doi.org/10.15649/cuidarte.1273 (c) $\bigodot_{\mathrm{BY}}$ E-ISSN: 2346-3414

(1) Jorge Homero Wilches Visbal

(1) Midian Clara Castillo Pedraza ${ }^{2}$

(1) Xiomara Zilena Serpa Romero ${ }^{3}$

1 Programas de Odontología y Medicina, Facultad de Ciencias de la Salud, Universidad del Magdalena, Santa Marta, Colombia. E-mail: jhwilchev@gmail.com

2 Programa de Odontología, Facultad de Ciencias de la Salud, Universidad del Magdalena, Santa Marta, Colombia. E-mail: midianclar@ gmail.com

3 Programa de Odontología, Facultad de Ciencias de la Salud, Universidad del Magdalena, Santa Marta, Colombia. E-mail: xiomaraserpa@gmail.com

Señora Editora,

La enfermedad por coronavirus 2019 (COVID-19), una afección respiratoria aguda causada por el SARS-CoV2, ha sido clasificada como pandemia por la Organización Mundial de la Salud (OMS) una vez que se ha expandido a 215 países del mundo, ha infectado a más de 7.800 .000 personas y cerca de 440.000 personas han muerto por su causa'.

El SARS-CoV2 es un coronavirus tipo $\beta$ de características genómicas similares al MERS-CoV y al SARS-CoV1 los cuales

Debido a la alta tasa de contagio y propagación del SARS-CoV2, diversas medidas de mitigación han sido empleadas. afectaron a más de 10.000 personas en las últimas dos décadas ${ }^{2,3}$. Debido a la alta tasa de contagio y propagación del SARS-CoV2, diversas medidas de mitigación han sido empleadas. Entre las medidas adoptadas se encuentran la cuarentena, el distanciamiento físico, la limpieza de superficies y aerosoles, así como el uso de equipos de protección personal (EPP) ${ }^{2-6}$.

Dado que no se dispone de vacuna y tratamientos suficientemente efectivos, evitar el contagio por contacto directo con superficies, aerosoles o suspensiones contaminadas con el SARS-CoV2 es la primera y más importante medida de contención contra la pandemia ${ }^{7}$. Esto obedece a que el SARS-CoV2 puede sobrevivir

Recibido: mayo 29 de 2020 Aceptado: julio 10 de 2020

Publicado: noviembre 13 de 2020 $\nabla{ }^{*}$ Correspondencia Jorge Homero Wilches Visbal E-mail:jhwilchev@gmail.com 
por $3 \mathrm{~h}$ en aerosoles, $4 \mathrm{~h}$ en superficies de cobre, un día en cartón, dos días en acero inoxidable y hasta 3 días en plástico ${ }^{8}$. Dada la alta demanda de los EPP, que provoca escasez y obliga a la reutilización de estos ${ }^{7,9}$, métodos eficaces de limpieza son cruciales.

Hasta ahora, los agentes germicidas potencialmente aplicables en la lucha contra la COVID-19 se proponen sobre la base de que su efectividad ha sido comprobada en varios tipos de patógenos, especialmente, en aquellos que guardan semejanza genética con el SARS-CoV2 $2^{7,10,11}$. Los agentes germicidas, sobre los que existe evidencia fehaciente, pueden ser clasificados en químicos, físicos y térmicos.

Los agentes químicos involucran una solución heterogénea capaz de desintegrar la membrana lipídica del coronavirus. Soluciones jabonosas aplicadas durante 20 - $30 \mathrm{~s}$, o aquellas con 0,1\% de hipoclorito de sodio o 62 - 71\% de alcohol y/o 0,5\% de peróxido de hidrógeno empleadas durante $1 \mathrm{~min}$, han mostrado su efectividad contra varios tipos de coronavirus ${ }^{7}$. Los agentes químicos son los germicidas más difundidos, accesibles y utilizados para evitar el contagio.

Los agentes físicos son aquellos que utilizan radiación electromagnética capaz de inducir modificaciones fotoquímicas al material genético (ARN) de los coronavirus, inhibiendo de este modo, sus mecanismos replicativos. La radiación ultravioleta, un tipo de radiación electromagnética con longitud de onda entre los 400-100 nm del espectro electromagnético y la radiación gamma, un tipo de radiación ionizante con longitud de onda inferior a 1 pm, son dos de los principales agentes físicos.

La luz ultravioleta tipo C (UV-C) lejana (207-222 nm) aplicada con una irradiancia de $2 \mathrm{~mJ} / \mathrm{cm}^{2}$ ha conseguido inactivar el virus de la influenza suspendido en aire ${ }^{11}$. En un artículo reciente ${ }^{12}$, se halló que la UV-C lejana de $222 \mathrm{~nm}$ con intensidad de $3 \mathrm{~mJ} / \mathrm{hcm}^{2}$ irradiando durante $25 \mathrm{~min}$ aproximadamente, podría inactivar varios tipos de coronavirus en aerosol sin causar daños en el cuerpo humano, siendo esto último una diferencia esencial con respecto a la UV-C germicida común de 254 o $265 \mathrm{~nm}^{11,13}$. La utilización de UV-C germicida de $265 \mathrm{~nm}$, con irradiancias entre 1 y 4,32 J/ $\mathrm{cm}^{2}$ parece ser una herramienta idónea para descontaminar máscaras quirúrgicas o N95 y protectores faciales de plástico (visores) en cabinas de bioseguridad ${ }^{13}$. A pesar de la demostrada ventaja de la UV-C lejana de 222 $\mathrm{nm}$, debe advertirse que aún se desconocen sus efectos estocásticos (de largo plazo).

Las máscaras N95 y quirúrgicas deben reservarse para el personal sanitario ocupacionalmente expuesto mientras que las máscaras de tela son indicadas para el resto de la población ${ }^{9,14}$. En situaciones de pandemia, por la escasez de los EPP, es recomendable implementar prácticas que permitan el uso extendido y/o la reutilización limitada, en ese orden de prioridad, de respiradores de máscaras quirúrgicas o N95, siempre que no se hayan contaminado, ${ }^{9}$. En este sentido, la descontaminación con UV-C de EPP, bajo las irradiancias y/o intensidades mencionadas, podría disminuir el contagio sobre la base de que algún grado de descontaminación siempre será preferible a la reutilización de máscaras sin descontaminar ${ }^{13}$. En suma, la radiación UV-C no solo permitiría desinfectar superficies y EPP (254 o $265 \mathrm{~nm}$ ), como los agentes químicos, sino que también parece ser una herramienta prometedora para inactivar el SARS-CoV2 en aerosoles, 
incluso, en lugares públicos o cerrados con presencia de personas $(222 \mathrm{~nm})^{11,12}$.

La radiación gamma es una herramienta ampliamente aceptada para la esterilización de equipos de protección personal contaminados con bacterias y virus. Sin embargo, se ha encontrado que a la dosis de radiación utilizada para inactivar virus y bacterias (24 kGy), la capacidad de filtrado de las máscaras ha sido desmejorada significativamente $^{16}$. Además, su uso no es apto para lugares públicos por el conocido detrimento a la salud humana que causaría una dosis tan alta o la aplicación continua de radiación $\mathrm{X}$, aunque se usara una dosis o energía menor.
La sinergia entre la aplicación de UV-C lejana de 222 nm (por su efectividad para eliminar el coronavirus y su inocuidad, al menos a corto plazo, para el ser humano) y soluciones basadas en alcohol al 70\% (por su eficacia para inactivar al coronavirus en superficies, su practicidad y bajo costo) sería una medida de contención que merece ser explorada y validada

Los agentes térmicos actúan desnaturalizando las proteínas de la nucleocápside del coronavirus, mediante la aplicación de calor. Se ha encontrado evidencia de que un protocolo térmico de $80^{\circ} \mathrm{C}$ durante $1 \mathrm{~min}$ es efectivo para reducir considerablemente la actividad de varios tipos de coronavirus en suspensión10. Para el SARS-CoV1, se observó pérdida de integridad cuando sometido a una temperatura de $53^{\circ} \mathrm{C}$ por $10 \mathrm{~min}$. En un estudio reciente ${ }^{17}$, se determinó que un protocolo de $92^{\circ} \mathrm{C}$ con 15 min inactiva totalmente el SARS-CoV2. Como no se conocen los cambios estructurales que pueden inducir estos protocolos térmicos en los distintos tipos de máscaras, cada institución deberá evaluar combinaciones de temperatura y tiempo para impedir la pérdida de funcionalidad ${ }^{10}$.

De acuerdo con lo expuesto, consideramos que la sinergia entre la aplicación de UV-C lejana de $222 \mathrm{~nm}$ (por su efectividad para eliminar el coronavirus y su inocuidad, al menos a corto plazo, para el ser humano) y soluciones basadas en alcohol al 70\% (por su eficacia para inactivar al coronavirus en superficies, su practicidad y bajo costo) sería una medida de contención que merece ser explorada y validada a fin de transitar prontamente hacia un desconfinamiento seguro.

Conflicto de intereses: ninguno declarado.

\section{Referencias}

1. Colombia. Ministerio de Salud y Protección Social. Reporte Mundial y Nacional de Casos por COVID-19 [Internet]. 2020 [citado 16 de junio de 2020]. Disponible en: https://twitter.com/MinSaludCol/status/1272648258435891202

2. Fernandez D, Alonso LM, Fernández JA, Ordás B MS. Todo lo que necesitas saber del Coronavirus. Tiempos enfermería y salud [Internet]. 2019;2(7):1-10. Disponible en: https://tiemposdeenfermeriaysalud.es/journal/article/view/73/60

3. Fallahi HR, Keyhan SO, Zandian D, Kim S-G, Cheshmi B. Being a front-line dentist during the Covid-19 pandemic: a literature review. Maxillofac Plast Reconstr Surg. 2020;42(1):1-9. https://doi.org/10.1186/s40902-020-00256-5

4. Güner R, Hasanoğlu i, Aktaş F. Covid-19: Prevention and control measures in community. Turkish J Med Sci. 2020;50(SI-1):571-7. https://doi.org/10.3906/sag-2004-146

5. Peng X, Xu X, Li Y, Cheng L, Zhou X, Ren B. Transmission routes of 2019-nCoV and controls in dental practice. Int J Oral Sci [Internet]. 3 de diciembre de 2020;12(1):1-9.

https://doi.org/10.1038/s41368-020-0075-9 
6. Balachandar V, Mahalaxmi I, Kaavya J, Vivekanandhan G, Ajithkumar S, Arul N, et al. COVID-19: Emerging protective measures. Eur Rev Med Pharmacol Sci. 2020;24(6):3422-5.

7. Kampf G, Todt D, Pfaender S, Steinmann E. Persistence of coronaviruses on inanimate surfaces and their inactivation with biocidal agents. J Hosp Infect [Internet]. 2020;104(3):24651. https://doi.org/10.1016/j.jhin.2020.01.022

8. Van Doremalen N, Bushmaker T, Morris DH, Holbrook MG, Gamble A, Williamson BN, et al. Aerosol and Surface Stability of SARS-CoV-2 as Compared with SARS-CoV-1 [Internet]. The New England journal of medicine. 2020. p. 1-4. https://doi.org/10.1101/2020.03.09.20033217

9. Center for Disease Control and Prevention (CDC). Recommended Guidance for Extended Use and Limited Reuse of N95 Filtering Facepiece Respirators in Healthcare Settings [Internet]. 2020 [citado 16 de junio de 2020]. Disponible en:

https://www.cdc.gov/niosh/topics/hcwcontrols/recommendedguidanceextuse.html

10. Kampf G, Voss A, Scheithauer S. Inactivation of coronaviruses by heat. J Hosp Infect [Internet]. Disponible en:

https://www.journalofhospitalinfection.com/article/S0195-6701(20)30124-9/fulltext

11. Welch D, Buonanno M, Grilj V, Shuryak I, Crickmore C, Bigelow AW, et al. Far-UVC light: A new tool to control the spread of airborne-mediated microbial diseases. Sci Rep [Internet]. 2018;8(1):1-7. https://doi.org/10.1038/s41598-018-21058-w

12. Buonanno M, Welch D, Shuryak I, Brenner DJ. Far-UVC light efficiently and safely inactivates airborne human coronaviruses. Res Sq [Internet]. 2020.

https://doi.org/10.21203/rs.3.rs-25728/v1

13. Theory Division CCLRI, Card KJ, Crozier D, Dhawan A, Dinh M, Dolson E, et al. UV Sterilization of Personal Protective Equipment with Idle Laboratory Biosafety Cabinets During the Covid-19 Pandemic. medRxiv [Internet]. 2020;2020.03.25.20043489. Disponible en: https://www.medrxiv.org/content/10.1101/2020.03.25.20043489v1\%0Ainternal-pdf://0.0.23.4/2020.03.25.html

14. Center for Disease Control and Prevention (CDC). Use of Cloth Face Coverings to Help Slow the Spread of COVID-19 [Internet]. 2020 [citado 16 de junio de 2020]. Disponible en: https://espanol.cdc.gov/coronavirus/2019-ncov/prevent-getting-sick/cloth-face-cover.html?fbclid=IwAR0yZorXdc1BAEcG9kPErLFzTvr1_CAtdwcdajQ5STizoz9r8qT1wqorG3E

15. U.S. Food and Drug Administration (FDA). Surgical Mask and Gown Conservation Strategies - Letter to Health Care Providers [Internet]. 2020 [citado 16 de junio de 2020]. Disponible en: https://www.fda.gov/medical-devices/letters-health-care-providers/surgical-mask-and-gown-conservation-strategies-letter-health-care-providers

16. International Atomic Energy Agency (IAEA). Radiation Effective in Sterilizing Personal Protective Equipment Except For Respiratory Masks [Internet]. 2020 [citado 17 de mayo de 2020]. Disponible en: https://www.iaea.org/newscenter/pressreleases/radiation-effective-in-sterilizing-personal-protective-equipment-except-for-respiratory-masks-iaea

17. Pastorino B, Touret F, Gilles M, Lamballerie X De, Charrel RN. Evaluation of heating and chemical protocols for inactivating SARS-CoV-2. bioRxiv [Internet]. 2020;0-8.

https://doi.org/10.1101/2020.04.11.036855 